\title{
Velocity distribution of fluidized granular gases in the presence of gravity
}

\author{
J. Javier Brey and M. J. Ruiz-Montero \\ Física Teórica, Universidad de Sevilla, Apdo. de Correos 1065, E-41080 Sevilla, Spain
}

(Received 8 October 2002; published 27 February 2003)

\begin{abstract}
The velocity distribution of a fluidized dilute granular gas in the direction perpendicular to the gravitational field is investigated by means of molecular dynamics simulations. The results indicate that the velocity distribution can be exactly described neither by a Gaussian nor by a stretched exponential law. Moreover, it does not exhibit any kind of scaling. In fact, the actual shape of the distribution depends on the number of monolayers at rest, on the restitution coefficient and on the height at what it is measured. The role played by the number of particle-particle collisions as compared with the number of particle-wall collisions is discussed.
\end{abstract}

DOI: 10.1103/PhysRevE.67.021307

PACS number(s): 45.70.Mg, 81.05.Rm, 47.20.-k

\section{INTRODUCTION}

Steady states of granular fluids require energy that is continuously supplied to the system, in order to compensate the energy loss in collisions. In many experimental situations, this is done by means of a vibrating wall. If the vibration is strong enough, the steady state will correspond to a completely fluidized situation. The velocity distribution function (VDF) of a granular fluid in such a steady state has attracted a lot of attention in recent years. It has been studied theoretically, numerically, and by means of experiments, using very different drivings of the system. It seems clear at this stage that the results are strongly dependent on the characteristics of the driving mechanism, and also on the granular fluid itself $[1,2]$. In this paper, we will be dealing with the VDF of a fluidized low density granular system in the presence of gravity, and in a situation when the number of wall-particle collisions is much smaller than the number of particleparticle collisions. In these conditions, one can expect that the VDF will be dominated by the intrinsic dynamics of the granular gas, and not by the peculiarities of the energy injection.

One of the first experimental studies of the VDF of a granular fluid in the situation described above was carried out by Warr et al. [3]. They considered a vertical twodimensional system of steel balls under vertical sinusoidal vibration, and found that the width of the VDF for the vertical $\left(v_{y}\right)$ and horizontal $\left(v_{x}\right)$ components of the velocity decreased as height increased, showing that the granular temperature was a decreasing function of height. Besides, the distribution for $v_{x}$ seemed to be quite well fitted by a Gaussian distribution. A similar result was found by Helal et al [4], who performed molecular dynamics simulations of a twodimensional system of hard disks, concluding again that the distribution function for $v_{x}$ was Gaussian, at least in the thermal velocity region. A few years later, Kudrolli and Henry [5] studied experimentally a system very similar to that in Ref. [3], but now the plane of the system formed a certain angle $\theta$ with the horizontal direction. By varying $\theta$, the external (gravitational) field was modified, allowing to study the effect of varying the particle-particle and particle-wall number of collisions ratio. As the steady state exhibited gradients in the vertical direction, they studied the VDF in a region chosen so that density varied weakly inside it. They found that the width of the distribution for $v_{x}$ increased with $\theta$, and that for the larger values of $\theta$ studied, the distribution was approximately Gaussian. The authors claimed that this happened because increasing $\theta$ increases the frequency of particle-wall collisions, resulting in broader distributions.

Rouyer and Menon [6] have reported the results of another experimental study of a two-dimensional system of steel balls in the presence of gravity. To avoid the effects of the inhomogeneities in the VDF, they placed the system between two identical vibrating walls, one located at the top and the other at the bottom of the system. For fast enough shaking, a symmetrical around the center of mass state was generated, with small gradients in the central region. The VDF was measured in that central region, and it was found that the distribution for the horizontal component of the velocity, when scaled with its width $\sigma_{x}$, showed an universal behavior. Moreover, it could be fitted in the whole velocity range by means of a stretched exponential type of distribution,

$$
R\left(c_{x}\right)=C^{-1} \exp \left\{-A\left|c_{x}\right|^{1.5}\right\}
$$

where $c_{x}=v_{x} / \sigma_{x}$, while $A$ and $C$ are constants that can be determined from the normalization condition and the fact that the second moment must be unity, resulting $A \simeq 0.797$, $C \simeq 2.101$. It must be noticed that, although the same type of behavior had been previously justified theoretically [7], it appeared in the context of uniformly heated systems, and was only displayed by the high-energy tails of the velocity distribution. In this sense, it is remarkable that, for all the values of the parameters considered by Rouyer and Menon, and in the whole velocity range analyzed, the Gaussian distribution provided always a worse fit to the experimental data than $R\left(c_{x}\right)$. On the other hand, previous studies had found a Gaussian behavior, at least for some range of the parameters [3-5], as mentioned above.

One of the most important results reported in Ref. [6] is the "universality" of $R\left(c_{x}\right)$, which was verified by the authors for quite a wide range of shaking intensities (temperatures) and number of particles (densities) of the system. They present this as a robust property. Nevertheless, recent experiments by Blair and Kudrolli [8] suggest that the scaled horizontal VDF of a vibrated granular system depends on the number of particles, and only when this number is small 
enough it can be well fitted by $R\left(c_{x}\right)$. It can be argued that the reason for this apparent contradiction is that the experimental setup was different in both cases and, in fact, inhomogeneities were stronger in the experiment by Blair and Kudrolli than in the one by Rouyer and Menon. Whether these inhomogeneities are enough to destroy the universal behavior found by Rouyer and Menon is not clear at the moment. Finally, Barrat and Trizac [9] studied by molecular dynamics simulations a system in conditions similar to those used by Rouyer and Menon, but in the absence of gravity. They found that the transversal VDF was well fitted by $R\left(c_{x}\right)$, and a slight dependence of this distribution on the parameters was reported.

In this paper, we will study the distribution function of the horizontal component of the velocity of a vibrated granular fluid in the presence of gravity by using molecular dynamics simulations. Computer simulations are a very useful tool to study the velocity distribution of a system, as they provide detailed information about positions and velocities of all particles of the system at any moment. The construction of the corresponding distributions is straightforward, and averages over different times and initial conditions provide the statistical accuracy needed to compute the tails of the distributions. The goal of this study is to clarify the behavior of the velocity distribution of a fluidized low density granular system in the presence of gravity, and, in particular, to investigate the existence of a universal scaling behavior, independently of the parameters of the system.

\section{MOLECULAR DYNAMICS SIMULATIONS}

We consider a two-dimensional system of $N$ inelastic hard disks of diameter $\sigma$ and mass $m$, whose collisions are characterized by a constant coefficient of normal restitution $\alpha$. There is a gravity field $\mathbf{g}=-g \mathbf{u}_{y}$, acting in the negative direction of the $y$ axis, and a vibrating wall located at the bottom of the system $(y=0)$. The system size in the $X$ direction is $S$, and periodic boundary conditions are employed in that direction. Besides, and unless otherwise indicated, the system is opened at the top. The vibrating wall is elastic, and moves in a sawtooth manner, i.e., all particles colliding with the wall find it moving upwards with velocity $v_{w}$. Moreover, the amplitude of the wall vibration will be considered so small as compared with the mean free path of the particles, that the position of the wall can be taken accurately as fixed at $y=0$. Although this is clearly an ideal approximation to the movement of a real vibrating wall, we will be studying the VDF in regions not too close to the wall, so the details of its movement are expected to be unimportant, as long as we stay in a regime in which no waves are generated in the system [1].

The described system exhibits a steady state with gradients only in the direction of gravity which has been well characterized in the low density limit [10] by using a hydrodynamic description. This steady state depends both on $\alpha$ and $N_{y}=N / S$, which is proportional to the number of monolayers in the direction of gravity at rest. It must be pointed out that this state is not always stable, and inhomogeneities appear in the transversal direction if the system is wide

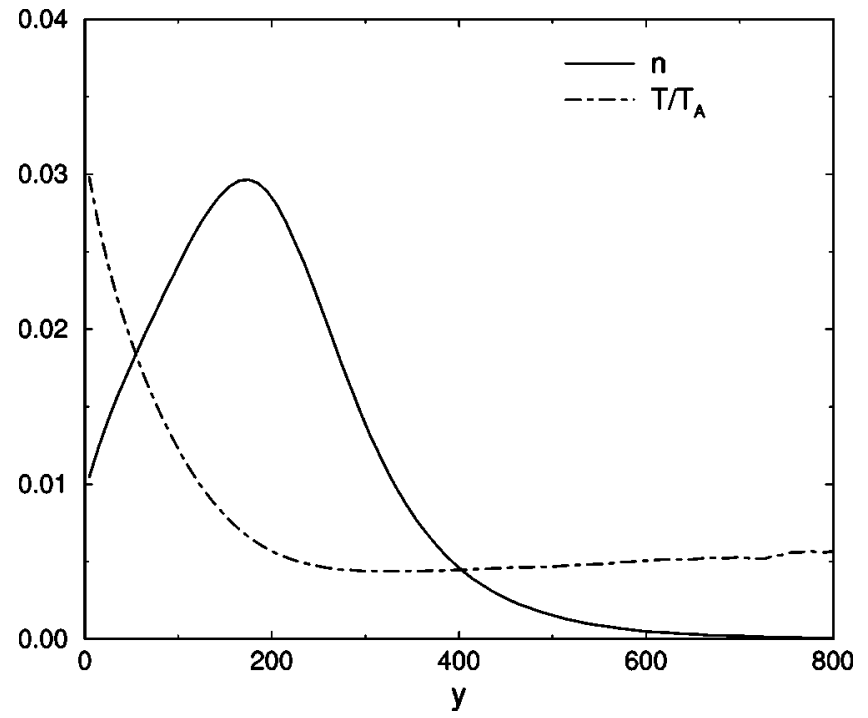

FIG. 1. Steady density (solid line) and temperature (dotteddashed line) profiles for a system with $\alpha=0.95$ and $N_{y}=6$. The temperature has been scaled with some arbitrary value, while density and height are measured in the dimensionless units defined in the main text.

enough, both with and without gravity [11-13]. In this work, we are interested in the VDF of the transversally homogeneous state, so the parameters will be chosen to ensure that inhomogeneities do not show up in the $X$ direction.

The evolution of the system was followed by using standard event driven molecular dynamics simulations [14], for several values of the parameters. In particular, the coefficient of normal restitution was always in the range $\alpha \geqslant 0.9$, which contains the value $\alpha=0.92$ characteristic of steel balls used in most of the mentioned experiments. The system width $S$ was given a value $S=50 \sigma$, which was small enough as to ensure homogeneity in the direction perpendicular to gravity for the values of $\alpha$ considered. The gravity field was set to $g=1$, while $m$ defines the unit of mass and $\sigma$ defines the unit of length. The velocity of the vibrating wall was chosen in each case such that the number density $n$ was always bellow a small enough value, $n \leqslant 0.15$, even in the denser regions of the system. Finally, for every value of $\alpha$, several different values of the number of particles were considered, in order to study its possible influence on the VDF. Let us notice that, as the system width $S$ is fixed, changing the number of particles is equivalent to changing the number of monolayers $N_{y}$, which is the relevant parameter.

The simulations started from an arbitrary initial situation that was left to evolve until the system reached a steady state. Typical density and temperature profiles in the steady state are shown in Fig. 1. Although the details of the profiles depend on the particular values of the parameters of the system, in all the cases reported here the density goes through a maximum at a certain height, while the temperature reaches a minimum, increasing from there on. It is important to notice that the temperature minimum does not coincide with the density maximum, and that the condition of small density gradient in a given region of the system does not imply small temperature gradient in it at all. In order to avoid the effect 
of inhomogeneities in the VDF, we have considered narrow layers perpendicular to the direction of the gradients at different positions throughout the system. The height of the cells $\Delta y_{c}$ was chosen so that the variation of density and temperature within them were negligible. For instance, in the case displayed in Fig. 1, the value $\Delta y_{c}=5$ was used. Cells at different positions will be considered to get information about the velocity distribution throughout the whole system. The distribution function for $v_{x}, P_{x}\left(v_{x} ; y\right)$ in each cell is constructed by sampling the velocities of particles inside it at different times, once the system was in the steady state. Besides, also different trajectories starting from different initial conditions were considered in the average process, in order to improve the statistics.

As the system is inhomogeneous in the $Y$ direction, $P_{x}\left(v_{x} ; y\right)$ is expected to depend on height $y$. Previous studies suggest that this dependence occurs only through the second moment of the distribution, but this is a result that has not been proved up to now for vibrated granular systems in the presence of gravity. We then define a scaled velocity by

$$
c_{x}=\frac{v_{x}}{\left\langle v_{x}^{2}\right\rangle^{1 / 2}},
$$

and a scaled velocity distribution

$$
\phi_{x}\left(c_{x} ; y\right)=\left\langle v_{x}^{2}\right\rangle^{1 / 2} P_{x}\left(v_{x} ; y\right) .
$$

If all the dependence of the distribution on height occurs through the second moment (i.e., the temperature), $\phi_{x}$ must be independent on height. This is one of the points to be checked in the simulations.

\section{SCALED VELOCITY DISTRIBUTION}

In Figs. 2 and 3 the scaled velocity distribution $\phi_{x}$ for $\alpha=0.95$ and two different values of the number of particles, $N=300\left(N_{y}=6\right)$ and $N=600\left(N_{y}=12\right)$, is plotted. The different symbols correspond to different heights in the system, as indicated in the figures. The solid line is the Gaussian distribution, while the dotted-dashed line is the stretched exponential $R\left(c_{x}\right)$ defined in Eq. (1). The layers whose distribution is displayed in the figures are located before the density maximum, at the maximum, and after it. The position of the density maximum is $y \simeq 100$ for both number of particles. The reason to display both $\phi_{x}$ and its logarithm is that the former provides visual information about the distribution for thermal velocities, while the latter is more convenient to identifying the behavior of the tails of the distribution.

The first point we want to investigate is whether the spatial dependence of the velocity distribution can be scaled out with its (local) second moment. If we consider the natural plot of $\phi_{x}$, the answer to this question may seem affirmative, at least in very good approximation. For given values of the parameters of the system, the symbols corresponding to different heights fall onto the same curve. Nevertheless, if we pay attention to the tails of the distribution, the answer is not so clear. Closer inspection of the logarithmic plots of $\phi_{x}$ shows that the scaling is not exact, and, in particular, the
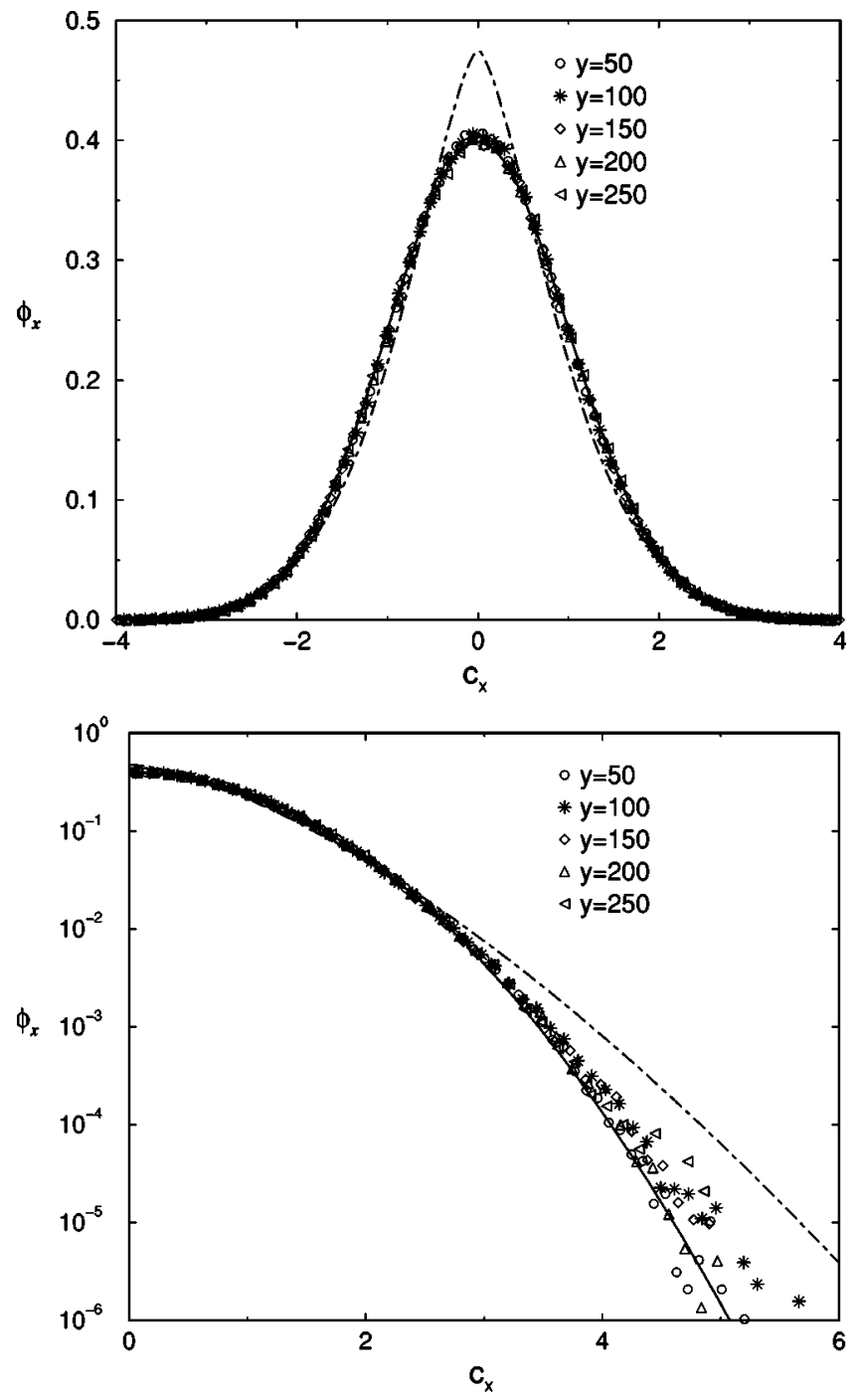

FIG. 2. Scaled velocity distribution at different heights for $\alpha$ $=0.95$ and $N_{y}=6$ (the same values of Fig. 1). The symbols are from the simulations, the continuous line is for a Gaussian distribution, and the dotted-dashed line is for the stretched exponential $R\left(c_{x}\right)$.

distribution corresponding to the layer located at the density maximum has always the most populated tail and, therefore, is the narrowest distribution. This implies that the height dependence of the distribution has not been completely scaled out. We have verified that this behavior is robust, in the sense that it is not the effect of a poor statistics in the lower density regions. Besides, the differences in shape of $\phi_{x}$ at different heights cannot be a consequence of averaging in space an inhomogeneous distribution, since the height of the layers is very small, as indicated above. Still, it could happen that the deviations from the scaling behavior were only relevant in a narrow region around the density maximum. To investigate whether this is the case, and also to provide a more quantitative measurement of the departure from the scaling, we have computed the scaled fourth moment of the distribution, $\gamma_{4}$, defined as 

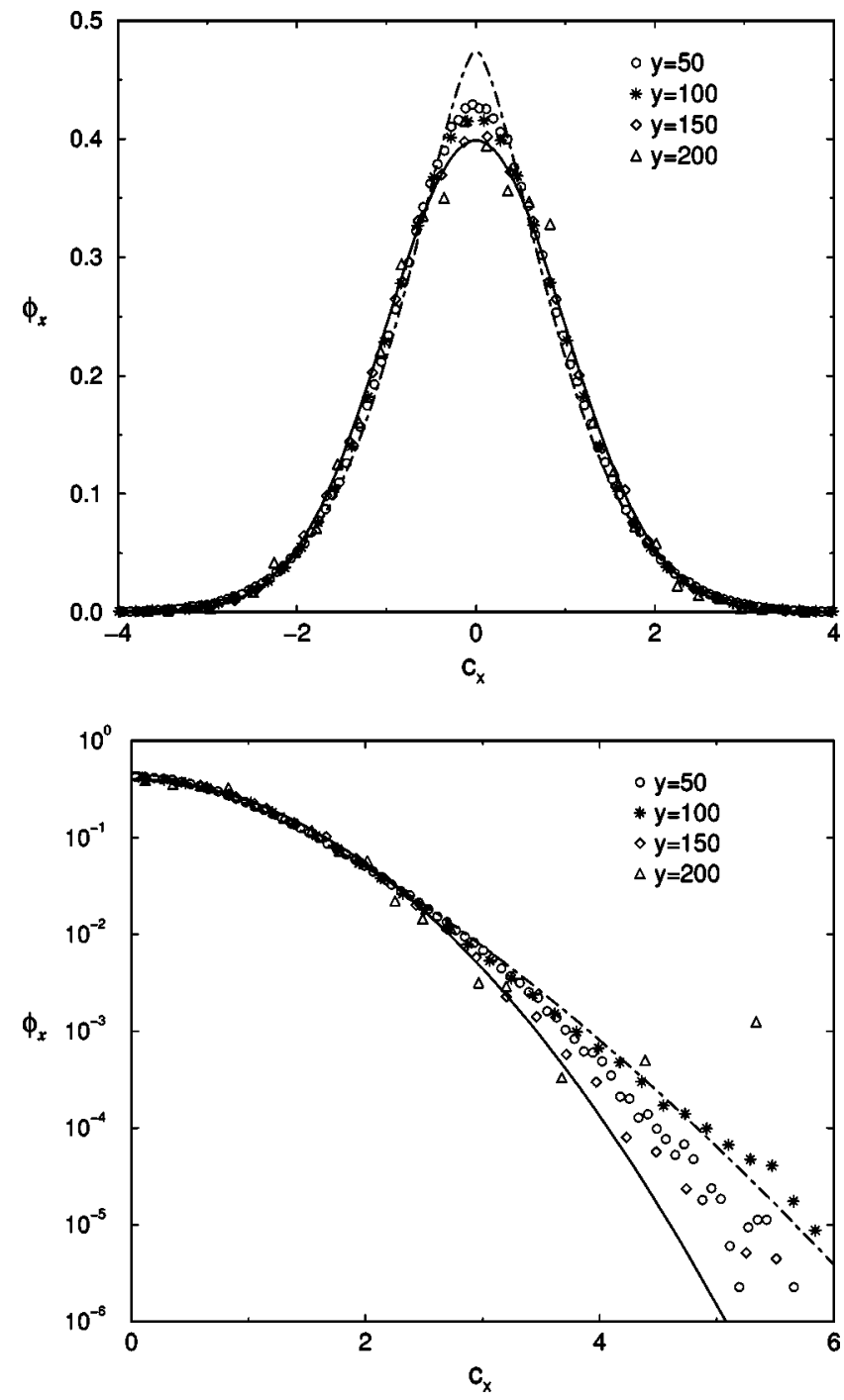

FIG. 3. The same as Fig. 2 but for $\alpha=0.95$ and $N_{y}=12$. The layer at $y=100$ corresponds to the density maximum, which is $n$ $=0.15$ in this case.

$$
\gamma_{4}=\frac{\left\langle v_{x}^{4}\right\rangle}{\left\langle v_{x}^{2}\right\rangle^{2}}
$$

If the velocity distribution depended on height only through its second moment, $\gamma_{4}$ would be constant through the system. If, in addition, the velocity distribution were a Gaussian, $\gamma_{4}=3$, while if it were given by the stretched exponential $R\left(c_{x}\right), \gamma_{4} \simeq 3.756$.

In Figs. 4 and $5 \gamma_{4}$ has been plotted as a function of height for $\alpha=0.95$ and $\alpha=0.9$, respectively. In each case, several values of the number of particles in the system have been considered. The range of $y$ plotted corresponds to the relevant region in the system, i.e., before the density decays to a very low value. In all cases, $\gamma_{4}$ exhibits a clear dependence on height. For given $\alpha$, the failure of the scaling is more evident the larger the number of particles in the system. In fact, it is the number of particles and not the density the parameter governing the behavior of the VDF, as far as the

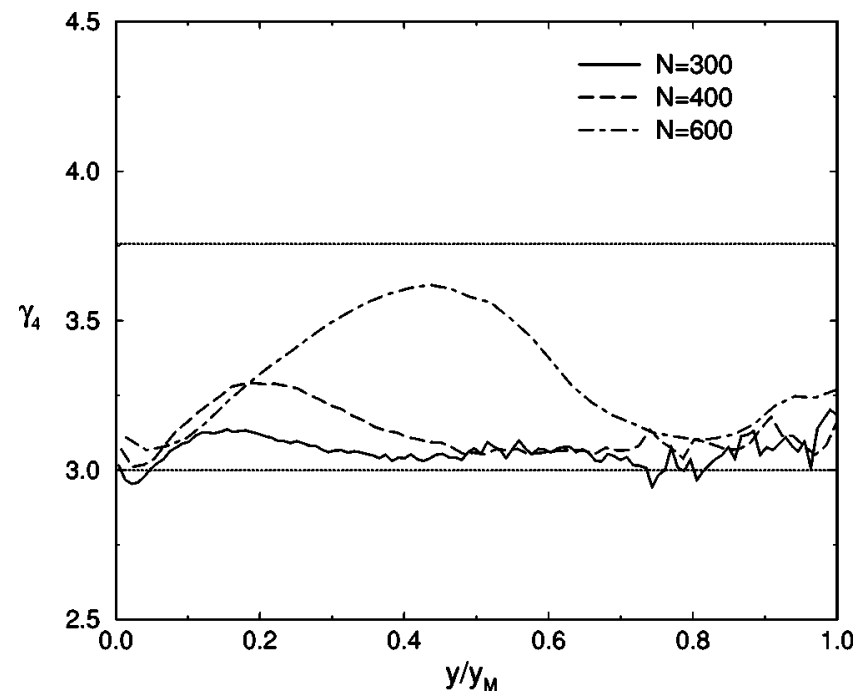

FIG. 4. Scaled fourth moment as a function of height for $\alpha$ $=0.95$ and three different values of the number of monolayers. The height has been divided by $y_{M}$, which is defined in each case as a height such that the density has decayed to a very low value.

system is fluidized. This has been checked by changing $v_{w}$ for fixed $\alpha$ and $N$, which implies modifying the density of the system, since it is opened at the top. The curves obtained for $\gamma_{4}$ were identical if the height was properly scaled with the vibrating velocity being used. The quantity $\gamma_{4}$ always follows the same trend: it takes a value very close to the Gaussian one in the vicinity of the vibrating wall, then passes trough a maximum, and decays to a more or less constant value that depends on both $\alpha$ and $N$. Comparison of the behavior of $\gamma_{4}$ with the density and temperature profiles in each case shows that the position of the maximum of $\gamma_{4}$ is close to the density maximum, while the region with a roughly constant value of the scaled fourth moment occurs beyond the temperature minimum. It must be noticed as well that, for the largest number of monolayers considered, $\gamma_{4}$

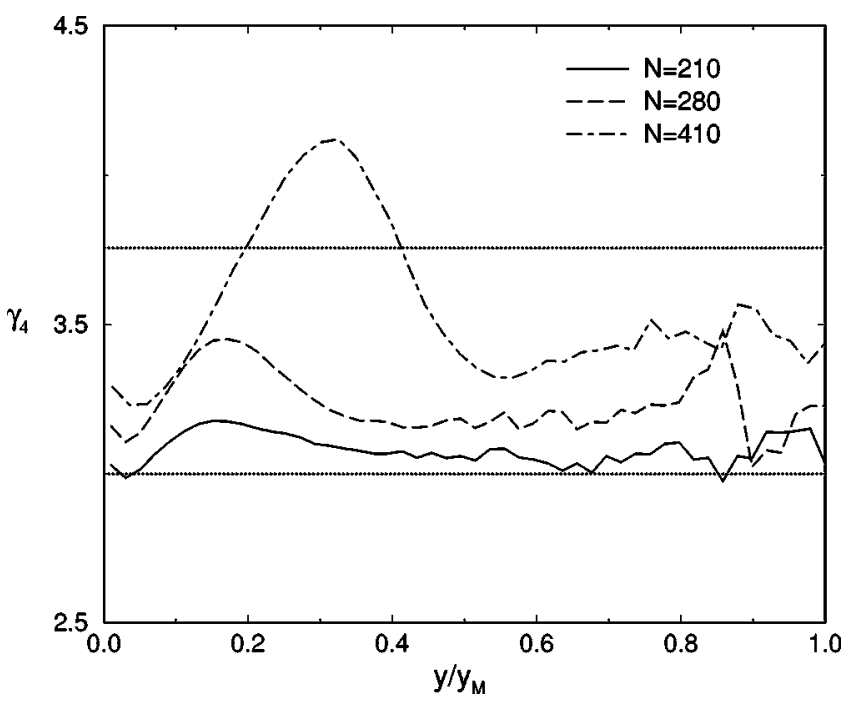

FIG. 5. The same as Fig. 4 but for $\alpha=0.9$. 
seems to increase for large heights. Although it is true that the statistical noise there is large, increasing the statistics does not change the observed behavior.

The first conclusion emerging from the simulations is that the dependence on height of the transversal VDF of a vibrated granular gas in the presence of gravity cannot be scaled out with its width, i.e., with the local transversal granular temperature of the system. This might appear as rather surprising, especially taking into account what happens in other states of a granular gas. For instance, in the homogeneous cooling state, the VDF depends on time, but this dependence can be completely scaled out with the temperature, and the scaled fourth velocity moment reaches a plateau after a transient period [15]. On the other hand, it is true that, for the cases reported here, the scaling can be taken as an accurate approximation in the thermal velocity region, at least for a not too large number of particles in the system. Besides, the more inelastic the system, the smaller the number of monolayers for which deviations from scaling begin to be significant.

Figures 2-5 also indicate that, even if we neglect the height dependence, the shape of the distribution still depends both on the inelasticity and the number of monolayers. For instance, in the case of Fig. 2, the VDF is very well approximated by a Gaussian distribution for velocities up to $\left|c_{x}\right|$ $\sim 4$. Some deviations from the Gaussian appear for large velocities, but they are not very significant. As the number of particles is increased, keeping $\alpha$ fixed, the deviations from the Gaussian shape become large, and the distribution gets narrower. This is the case of Fig. 3, where $\phi_{x}$ lies between the Gaussian distribution and $R\left(c_{x}\right)$ for thermal velocities, but the tail of the distribution is better approximated by the latter. If the number of particles is further increased, the distribution becomes narrower, in fact, narrower than $R\left(c_{x}\right)$. Again, as it happened with the scaling, the smaller $\alpha$, the smaller the number of particles for which deviations from the Gaussian distribution appear, as it follows from Figs. 4 and 5 .

Some authors have argued that, in a vibrated granular system, collisions with the wall tend to randomize the VDF, rendering it wide, while collisions between particles act as the ordering factor, because particle velocities are more parallel after collisions than before [5]. To check if this argument is consistent with our simulation results, we have computed $R_{p p / p w}$, the ratio between the number of particleparticle and particle-wall collisions. We found that, as expected, it is an increasing function of the number of monolayers in the system. For instance, for $\alpha=0.95$ we found $R_{p p / p w} \sim 41.1$ for $N=300, R_{p p / p w} \sim 67.5$ for $N=400$, and $R_{p p / p w} \sim 154.2$ for $N=600$. Since, as indicated above, the width of the distribution decreases as the number of particles increases, we conclude that the larger the $R_{p p / p w}$, the narrower the VDF. Moreover, the more inelastic the system, the more parallel the velocities after collisions, and the "ordering" mechanism is expected to be more efficient. This explains why, although for $\alpha=0.9$ and $N=410$ it is $R_{p p / p w}$ $\sim 103.3$, the distribution is in this case narrower than the narrowest reported here for $\alpha=0.95$, which corresponds to larger values of the ratio $R_{p p / p w}$.
Finally, the fact that the accuracy of the fit of the VDF to both $R\left(c_{x}\right)$ and the Gaussian distribution depends in most of the cases on the velocity interval considered, implies that $\phi_{x}$ cannot be fitted by a stretched exponential function $\phi_{x}$ $\sim \exp \left\{-C\left|c_{x}\right|^{\beta}\right\}$, with a single value of the exponent $\beta$, in the whole velocity range.

All the above discussion suggests, in our opinion, that the role played by the Gaussian distribution and $R\left(c_{x}\right)$ in the context of vibrated granular gases is very different. The Gaussian distribution is a limiting behavior for small enough number of particles and not too inelastic systems. As the number of particles in the system increases, the scaled velocity distribution becomes narrower. In this range of parameters, $R\left(c_{x}\right)$ is just a good fit valid in some cases, but it does not represent a universal behavior, not even a limiting behavior of the VDF for some well-defined conditions.

\section{SYMMETRIC BOUNDARY CONDITIONS}

Given that the conclusions reached up to now in this work are so different from those emerging from the experiments reported by Rouyer and Menon [6], it seems worth to simulate the same kind of boundary conditions they use, in order to discard that the discrepancies are due to peculiarities of the boundaries. Therefore, instead of considering just one vibrating wall at $y=0$, we have added another vibrating wall at $y=L=50 \sigma$. The choice of the maximum height $L$ of the system, was made as to obtain very similar system dimensions as those in Ref. [6]. The two vibrating walls are identical, and the vibrating velocity is chosen to generate symmetric around the center of the system stationary density and temperature profiles. The total number of particles was varied in the range $300 \leqslant N \leqslant 500$. In each case, we started from a homogeneous state, that was left to evolve until a stationary situation was reached. Then, the transversal VDF was computed in a layer located in the the center of the system, namely, between $0.4 \mathrm{~L}$ and $0.6 \mathrm{~L}$. Care was taken that the system remained in a fluidized state in that central region. Note that, in this way and as it was done in Ref. [6], the VDF is measured now in a quite wide region of the system, and not in a narrow layer as we did in the previous study of the open system. Even if the gradients in that central region are expected to be small, they will be nonvanishing, and the nonuniformity might influence the shape of the VDF.

In Fig. 6 we have plotted the scaled distribution $\phi_{x}$ for $\alpha=0.92$, which is roughly the value of the coefficient of restitution for the steel balls used in Ref. [6], and three different values of the number of particles. As it was the case for the open system, the scaled VDF is seen to depend on the number of particles, becoming narrower as $N$ increases. This can be interpreted again in terms of the role of the particleparticle versus particles-wall number of collisions, as also in this case the ratio $R_{p p / p w}$ is an increasing function of the number of monolayers. More specifically, we found from the simulation data that $R_{p p / p w} \sim 42$ for $N=300, R_{p p / p w} \sim 68$ for $N=400$, and $R_{p p / p w} \sim 103$ for $N=500$.

The relevant role of the stretched exponential $R\left(c_{x}\right)$ is also questioned by Fig. 6 . In all the cases displayed, the distribution for thermal velocities lies between the Gaussian and $R\left(c_{x}\right)$. The tail of the distribution gets closer to $R\left(c_{x}\right)$ 

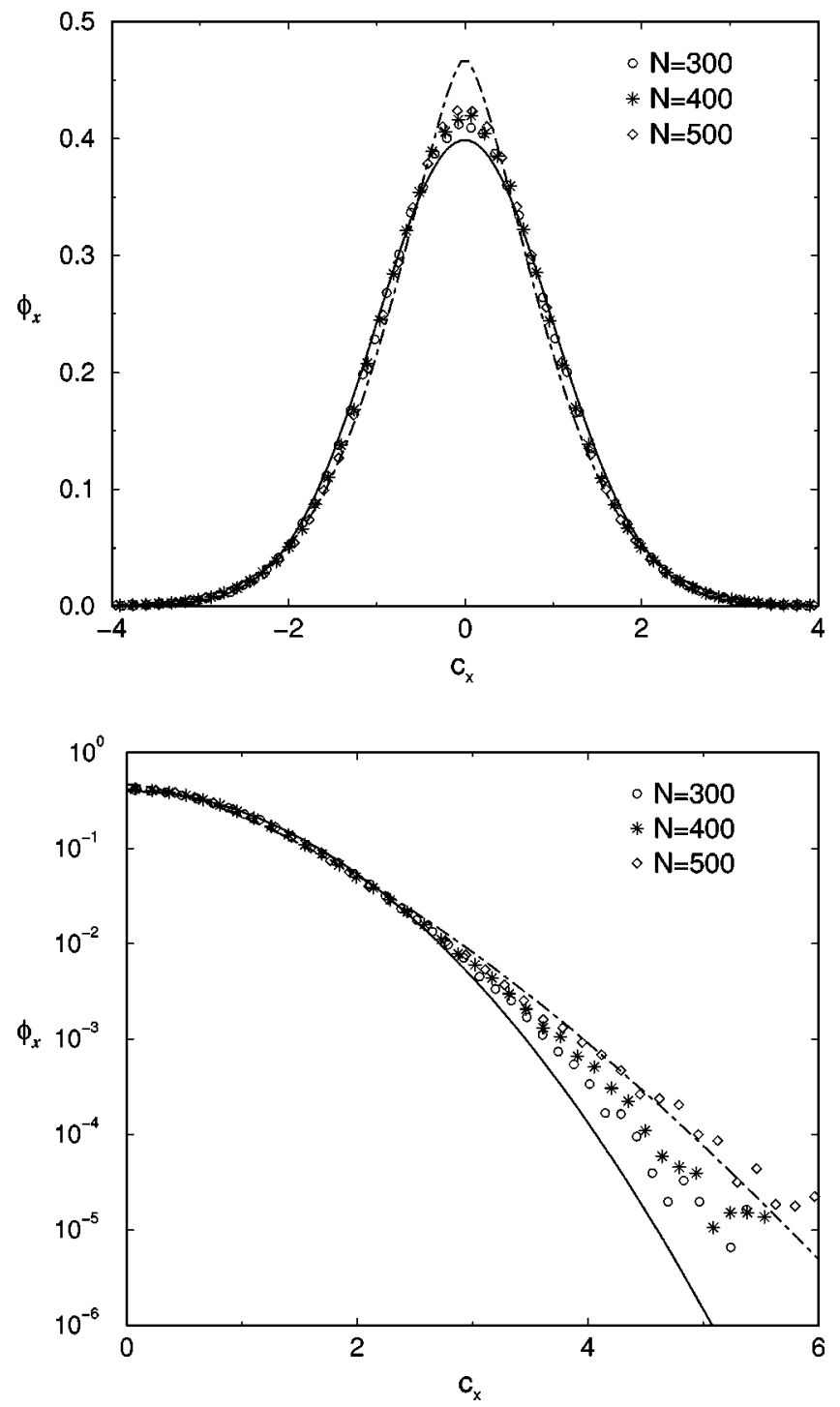

FIG. 6. Scaled velocity distribution at the central region of the system for symmetric boundary conditions, $\alpha=0.92$ and three values of the number of particles. The symbols are from the simulations, the continuous line is the Gaussian distribution, and the dotted-dashed line $R\left(c_{x}\right)$.

the larger $N$, and in fact only in the case $N=500$ is well fitted by the stretched exponential. Therefore, again the distribution cannot be fitted by a single stretched exponential in the whole velocity range. This is more clearly realized in Fig. 7, where $\ln \left[-\ln \left(\phi_{x} / \phi_{x}(0)\right]\right.$ has been plotted as a function of $\ln c_{x}$. Let us notice that, if the distribution were some general stretched exponential, $\phi_{x} \sim \exp \left\{-C\left|c_{x}\right|^{\beta}\right\}$, this plot would give a straight line whose slope would provide the exponent $\beta$. The symbols in the figure are from the simulations. Also plotted are two straight lines, one corresponding to $\beta=2$ (Gaussian behavior) and the other one to $\beta=1.5\left[R\left(c_{x}\right)\right]$ as a guide to the eye. A transition from Gaussian behavior for thermal velocities to a distribution with a smaller exponent $\beta$ for larger velocities is observed in all cases.

The results discussed up to now correspond to the VDF in the central region of the system. Nevertheless, we expect the

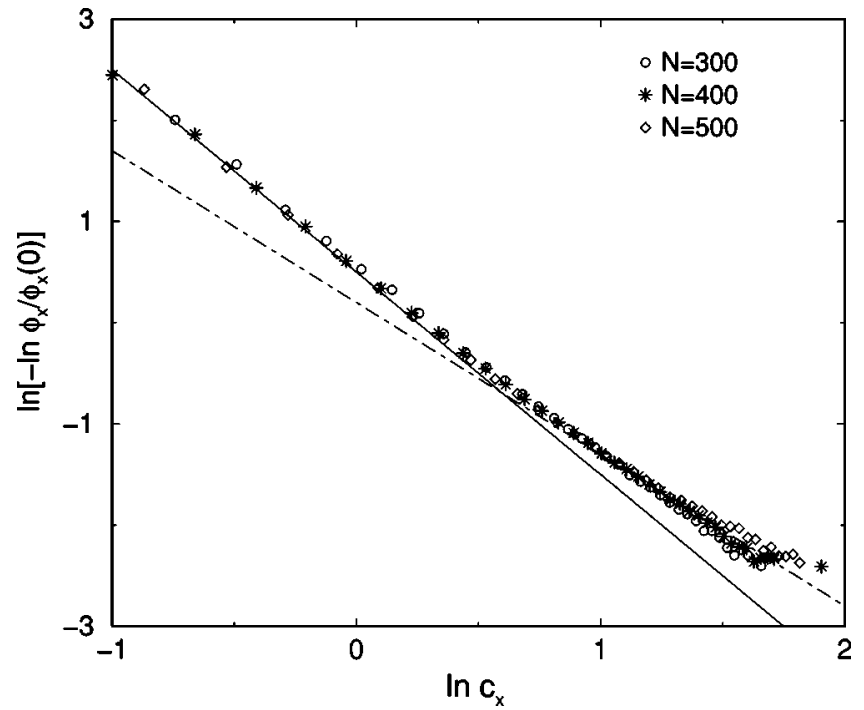

FIG. 7. The same as in Fig. 6, but using a double logarithmic plot. The solid line has the slope that corresponds to a Gaussian distribution, while the dotted-dashed line has the slope associated to $R\left(c_{x}\right)$. The positions of these two lines have been chosen quite arbitrarily in order to fit the maximum range of the simulation results.

transversal scaled VDF to depend also on $y$ in this case. In Fig. 8, $\gamma_{4}$ has been plotted as a function of height for the same values of the parameters as in Figs. 6 and 7. A clear dependence of the scaled moment of the transversal velocity distribution on height is observed. Again, we find that the dependence on height and the deviations from the Gaussian are larger, the larger the number of monolayers. As expected, $\gamma_{4}$ is symmetric around the center of mass. The exhibited double peak clearly indicated that the maximum of $\gamma_{4}$ does not happen either at the density maximum or at the temperature minimum, since they are both located at the center of the system.

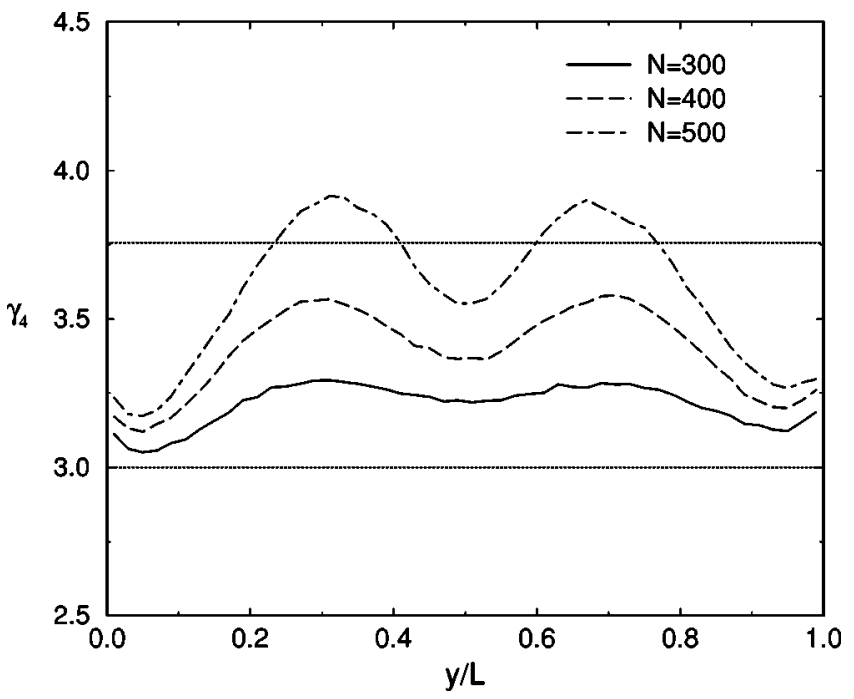

FIG. 8. Scaled fourth moment as a function of height in the symmetric case for the same values of the parameters as in Figs. 6 and 7. 


\section{CONCLUSIONS}

The transversal velocity distribution of a vibrated granular gas in the presence of gravity has been studied by using molecular dynamics simulations. It has been found that the shape of the distribution depends both on the inelasticity of the system and the number of monolayers in the direction of gravity. Besides, it depends on height, and this dependence cannot be fully scaled out with its width, i.e., with the local transversal granular temperature of the system. This contrasts with some previous reported results for granular gases under similar conditions. On the other hand, it is true that the scaling provides a quite accurate approximation valid for not too inelastic systems and for not too large number of monolayers at rest.

For a given value of the coefficient of restitution $\alpha$, the transversal velocity distribution becomes narrower as the number of monolayers in the system is increased. This can be understood by taking into account that increasing the number of monolayers increases the ratio of number of particle-particle collisions against particle-wall collisions. In a granular system, the particle-particle collisions tend to suppress "noise" in the velocity distribution, as the velocities after the collision are more parallel than before it. Then, it is sensible to expect that, the larger the relative number of particle-particle collisions, the narrower the velocity distribution. On the other hand, the more inelastic the system the more effective each collision in narrowing the velocity distribution.

The role played by the Gaussian distribution and the stretched exponential $R\left(c_{x}\right)$ has also been clarified. While the Gaussian is a limiting behavior shown in the quasielastic and small number of monolayers limit, $R\left(c_{x}\right)$ appears just as a good fit for the velocity distribution for some range of values of the parameters characterizing the system.

\section{ACKNOWLEDGMENTS}

We acknowledge support from the Ministerio de Ciencia y Tecnología (Spain) through Grant No. BFM2002-00303 (partially financed by FEDER funds).
[1] S. McNamara and J.L. Barrat, Phys. Rev. E 55, 7767 (1997).

[2] S. McNamara and S. Luding, Phys. Rev. E 58, 813 (1998).

[3] S. Warr, J.M. Huntley, and G.T.H. Jacques, Phys. Rev. E 52, 5583 (1995).

[4] K. Helal, T. Biben, and J.P. Hansen, Physica A 240, 361 (1997).

[5] A. Kudrolli and J. Henry, Phys. Rev. E 62, R1489 (2000).

[6] F. Rouyer and N. Menon, Phys. Rev. Lett. 85, 3676 (2000).

[7] T.C.P. van Noije and M.H. Ernst, Granular Matter 1, 57 (1998).

[8] D.L. Blair and A. Kudrolli, Phys. Rev. E 64, 050301 (2001).

[9] A. Barrat and E. Trizac, Phys. Rev. E 66, 051303 (2002).
[10] J.J. Brey, M.J. Ruiz-Montero, and F. Moreno, Phys. Rev. E 63, 061305 (2001).

[11] P. Sunthar and V. Kumaran, Phys. Rev. E 64, 041303 (2001).

[12] J.J. Brey, M.J. Ruiz-Montero, F. Moreno, and R. García-Rojo, Phys. Rev. E 65, 061302 (2002).

[13] E. Livne, B. Meerson, and P.V. Sasorov, Phys. Rev. E 65, 021302 (2002).

[14] M.P. Allen and D.J. Tildesley, Computer Simulations of Liquids (Clarendon Press, Oxford, 1987).

[15] J.J. Brey, M.J. Ruiz-Montero, and D. Cubero, Phys. Rev. E 54, 3664 (1996). 